\title{
Optic disc measurement with the Zeiss four mirror contact lens
}

\author{
A F Spencer, S A Vernon
}

\begin{abstract}
A knowledge of the optic disc size may be of value when assessing the glaucoma suspect. The vertical diameter of the optic disc was measured using a Zeiss four mirror gonioscope and a 900 Haag-Streit slit-lamp in one eye of 39 patients, 32 with refractive errors within 3 dioptres of emmetropia. The disc was measured by projecting a slit beam of known height onto the image of the disc. A magnification factor for the contact lens was calculated from first principles and disc height recalculated. These measurements were compared with those obtained by photographic methods using the corrections suggested by Bengtsson and Krakau. In the analysis on the 32 eyes within 3 dioptres of emmetropia the best correlation with clinical measurements was obtained with correction 3 using spectacle refraction and keratometry $(r=0.8614)$. The contact lens measurement was within plus or minus $0.1 \mathrm{~mm}$ of the photographic measurement in $67 \%$ of cases and plus or minus $0.2 \mathrm{~mm}$ in all cases. This simple method is advocated for the routine assessment of optic disc size.

(Br F Ophthalmol 1994; 78: 775-780)
\end{abstract}

A diagnosis of the presence of glaucomatous change in an optic disc requires the observer to determine one or more abnormalities in the appearance of the disc in question. ${ }^{1}$ Disc size is known to vary considerably, and hence a large cup may be normal in a large disc and even a small cup may be abnormal in a small disc. ${ }^{12} \mathrm{~A}$ knowledge of the dimensions of the optic disc is therefore of value to the clinical ophthalmologist.

Methods of measurement in vivo include fundus photography with planimetry, ${ }^{36}$ measurement of the aerial image formed by an indirect ophthalmoscope, ${ }^{7}$ and measurement of distances using scanning laser ophthalmoscopy. ${ }^{8}$

A contact lens with a flat front surface produces a virtual erect image of the fundus in the vitreous that can be viewed by the slit-lamp microscope. ${ }^{9}$ The relative magnification of the image is determined by the refractive index of the contact lens, the ocular dimensions, and the power of the natural lens if present.

The dimensions of the image of a retinal object - for example, the vertical diameter of the optic disc, can therefore be estimated by comparison with a narrow slit beam producéd by the slit-lamp in the image plane of the microscope.

In this study the vertical diameter of the optic disc measured by the above method using a Zeiss four mirror contact lens is compared with three planimetric methods recently described by Bengtsson and Krakau. ${ }^{10}$

\section{Materials and methods}

The optic discs of one eye of 39 patients were examined, 32 of which had a refractive error within plus or minus $3.0 \mathrm{D}$ best sphere of emmetropia. There were 15 normals, eight ocular hypertensives, and 16 glaucoma patients.

\section{MEASUREMENT WITH ZEISS FOUR MIRROR}

The pupil was dilated with $1 \%$ tropicamide and the eye anaesthetised with proxymetacaine $0.5 \%$ or amethocaine $1 \%$. A Zeiss four mirror gonioscope contact lens was then applied gently to the cornea, the fundal image being observed through the central viewing zone. The optic disc was defined as the area inside the white peripapillary scleral ring. The vertical diameter was defined as the distance from the edge of the nerve fibre rim at 12 o'clock to the edge of the nerve fibre rim at 6 o'clock.

A narrow vertical slit beam of light was reduced progressively in size from $5 \mathrm{~mm}$ until it was judged to correspond to the size of the disc. The beam height was then recorded, from the scale on the slit-lamp, by an assistant. The slit beam was then reset to $5 \mathrm{~mm}$ and the measurement repeated twice. The observer performing the measurements was thus unaware of the results until all three readings had been taken. As the slit-lamp beam height scale is calibrated in $0.1 \mathrm{~mm}$ steps, measurements were judged by the assistant to the nearest $0.1 \mathrm{~mm}, 0.05$ increments being 'rounded up'. From the three readings a mean was derived.

\section{SLIT BEAM CALIBRATION}

Calibration of the biomicroscope used for the study was performed in the following manner: a focused beam was projected onto a card on which were printed parallel lines at different distances apart. The distance between five pairs of lines ranging from 1-5 mm apart was measured with a micrometre screw gauge by two observers, the mean being taken as the true distance. The size of the slit beam was then adjusted to coincide with the distance between the two lines and this was read off the scale on the biomicroscope in a similar manner to that employed for the disc height.

\section{CALCULATION OF MAGNIFICATION FACTOR FOR}

ZEISS FOUR MIRROR

This was calculated from first principles.

Calculation of the focal point of the plano concave lens/cornea combination can be performed in two ways:

$$
1 / \mathrm{f}=(\mathrm{n}-1) / \mathrm{r}
$$


$\mathrm{r}=$ radius of curvature $=7 \cdot 7 \mathrm{~mm}$

$\mathrm{n}=$ index of refraction of $\mathrm{BK} 7$ of lens/index of refraction of cornea $=1 \cdot 519 / 1 \cdot 336$

hence

$$
\begin{aligned}
1 / \mathrm{f} & =0.01779 \\
\mathrm{f} & =56.20 \mathrm{~mm}
\end{aligned}
$$

This gives us a good estimate but it is more accurate to use the formula:

$$
\mathbf{n}^{1 / 1} \mathbf{1}^{2}=\left(\mathbf{n}^{1}-\mathbf{n}^{2}\right) / \mathbf{r}
$$

$1^{2}=$ distance to image (focal point of lens)

$\mathrm{n}^{1}=$ refractive index of media behind contact lens

(that is, cornea/aqueous) $=1 \cdot 336$

$\mathrm{n}^{2}=$ refractive index of $\mathrm{BK} 7=1 \cdot 519$

$r=$ radius of curvature of the lens $=7 \cdot 7 \mathrm{~mm}$

hence

$$
\begin{aligned}
1 \cdot 336 / 1^{2} & =(1 \cdot 336-1 \cdot 519) / 7 \cdot 7 \\
1^{2} & =-1 \cdot 336 / 0 \cdot 0238 \\
1^{2} & =-56 \cdot 13 \mathrm{~mm}
\end{aligned}
$$

The optical centre or principal plane of the plano concave contact lens when applied to the cornea is on the anterior surface of the cornea.

The principal plane of the biconvex crystalline lens is calculated knowing the power of the anterior $\left(F^{1}\right)$ and posterior $\left(F^{2}\right)$ surface of the lens.

$$
\mathrm{F}^{1}=(\mathrm{n}-1) / \mathrm{r}
$$

where

$$
\begin{aligned}
\mathrm{n} & =\text { difference in refractive index lens/ } \\
& \text { aqueous } \\
& =1 \cdot 416 / 1 \cdot 336 \\
\mathrm{r} & =10 \mathrm{~mm}(\text { Gullstrand model eye })
\end{aligned}
$$

therefore

$$
F^{1}=5 \cdot 988 \mathrm{D}
$$

and

$$
\mathrm{F}^{2}=(\mathrm{n}-1) / \mathrm{r}
$$

where

$$
\begin{aligned}
\mathrm{n} & =\text { ratio for refractive index lens/vitreous } \\
& =1 \cdot 416 / 1 \cdot 336 \\
\mathrm{r} & =6 \mathrm{~mm} \text { (Gullstrand model eye) }
\end{aligned}
$$

therefore

$$
\mathrm{F}^{2}=9.980 \mathrm{D}
$$

and the principal plane of the equivalent thin lens is $2.25 \mathrm{~mm}$ from the front surface of the lens.

The anterior surface of the lens is $3.6 \mathrm{~mm}$ from the anterior corneal surface in the average eye.

Hence the distance from the principal plane of the cornea $+\mathrm{CL}$ to the principal plane of the crystalline lens $=3 \cdot 6+2 \cdot 25=5 \cdot 85 \mathrm{~mm}=\mathrm{d}$ (see below).

Power of 'cornea $+\mathrm{CL}$ ' is $\mathrm{F}^{3}$

$$
\begin{aligned}
\mathbf{F}^{3} & =1 /-\mathrm{f} \\
& =1 /-56.13 \mathrm{~mm} \\
& =-17.8 \mathrm{D}
\end{aligned}
$$

The crystalline lens has an effective power of 19.1 D (Gullstrand's model eye), focal distance $\mathrm{f}=69.908 \mathrm{~mm}$.

The effective power of 'cornea $+\mathrm{CL}$ ' in plane of crystalline lens is $\mathrm{F}^{4}$

$$
\begin{aligned}
\mathrm{F}^{4} & =\mathrm{F}^{3} / 1-\mathrm{dF}^{3} \\
\mathrm{~d} & =\text { distance moved in } \mathrm{m}=0 \cdot 00585 \mathrm{~m} \\
\mathrm{~F}^{4} & =-17 \cdot 8 / 1-[-0 \cdot 00585(-17 \cdot 8)] \\
& =-17 \cdot 8 /(1-0 \cdot 104) \\
& =-19 \cdot 86 \mathrm{D}
\end{aligned}
$$

So overall power of crystalline and 'cornea $+\mathrm{CL}^{\prime}=19 \cdot 1-19 \cdot 86=-0 \cdot 76 \mathrm{D}$

Having calculated the power of the 'cornea $+C L$ ' in the plane of the crystalline lens and knowing the distance of the crystalline lens to the object we can calculate the distance, from the lens, to the image.

Axial length $=24 \cdot 4 \mathrm{~mm}$ (Gullstrand's model eye)

Distance from retina (object) to principal plane of lens

$$
=24 \cdot 4-5 \cdot 85 \mathrm{~mm}=18 \cdot 55 \mathrm{~mm}
$$

Using the formula

$$
1 / \mathrm{v}-1 / \mathrm{u}=1 / \mathrm{f}
$$

$\mathrm{v}=$ distance lens to image

$\mathrm{u}=$ distance lens to object

$$
1 / \mathrm{f}=-0 \cdot 76 \mathrm{D}
$$

then

$$
\begin{aligned}
1 / \mathrm{v}-(1 /-0.01855)= & -0.76 \\
1 / \mathrm{v}= & -0.76+(1 /-0.01855) \\
\mathrm{v}= & -18.29 \mathrm{~mm} \\
& \text { (that is, behind lens) }
\end{aligned}
$$

Magnification for the contact lens is calculated from:

$$
\begin{aligned}
& \mathrm{I} / \mathrm{O}=18 \cdot 29 / 18 \cdot 55 \quad \text { image size }=\mathrm{I} \\
& \mathrm{I}=0.985 \times \mathrm{O} \quad \text { object size }=\mathrm{O}
\end{aligned}
$$

therefore real size $=$ image size $/ 0 \cdot 985$

\section{PHOTOGRAPHIC CORRECTIONS}

The axial length and corneal curvature of the eyes was measured using calibrated instruments (Coopervision Ultrascan Digital A and Javal Schiotz keratometer) and spectacle refractions were performed by experienced optometric staff at a separate examination. Photographs of the optic discs were taken at the highest magnification, 30 degree setting, with a Topcon fundus camera. A camera constant for the camera used was calculated by the method described by Bengtsson and Krakau. ${ }^{10}$ The photographic slides of the optic discs were projected onto a screen and the optic disc vertical diameter (using the same criteria as described above) was measured by two independent observers and the mean reading taken. The magnification produced by the projector was calculated for each photograph by the two observers who measured the black to black distance on the transparency with a scientific ruler and the height of the total image produced on the screen. Magnification 'drift' was found never to exceed $1 \%$ throughout the study. The slides had been magnified 23.5 times. The estimates described in Bengtsson and Krakau's paper were then applied to the mean image height obtained, taking into account the camera constant, in order to give the actual size of the optic disc. All three estimations were applied, that is, using (a) axial length only, (b) spectacle correction only, and (c) using spectacle correction and keratometry. 
ANALYSIS

The clinical measurements recorded were corrected after the slit beam calibration and for the 'magnification factor' of the Zeiss four mirror. These results were then compared for individual eyes with the calculated vertical disc diameter obtained from the three photographic methods using scattergram plots from which regression equations could be derived. For intraobserver variation the coefficient of variation of the Zeiss four mirror measurements is calculated.

\section{Results}

MEASUREMENT OF OPTIC DISC BY ZEISS FOUR MIRROR CONTACT LENS

The size of the optic discs ranged from $1 \cdot 3$ to $2 \cdot 0$ $\mathrm{mm}$ with a mean of $1.687(\mathrm{SD} 0 \cdot 194) \mathrm{mm}$.

\section{MEASUREMENT OF OPTIC DISC HEIGHT FROM THE} PROJECTED SLIDES

There was good agreement between observers $A$ and $B$ measuring the projected image of the optic discs. Figure 1 shows the actual measurements for these. Agreement was examined by plotting the difference between the two measurements

Figure 1 Screen measurements of the optic disc from projected slides (in $\mathrm{cm}$ ). The line of identity is shown.

Figure 2 Difference between observers $A$ and $B$ versus mean optic disc measurement from projected slides (in $\mathrm{cm}$ ).

Figure 3 Photographic estimate 1 (Bengtsson and Krakau) versus the mean of the clinical measurements with the contact lens. The line of identity is shown.
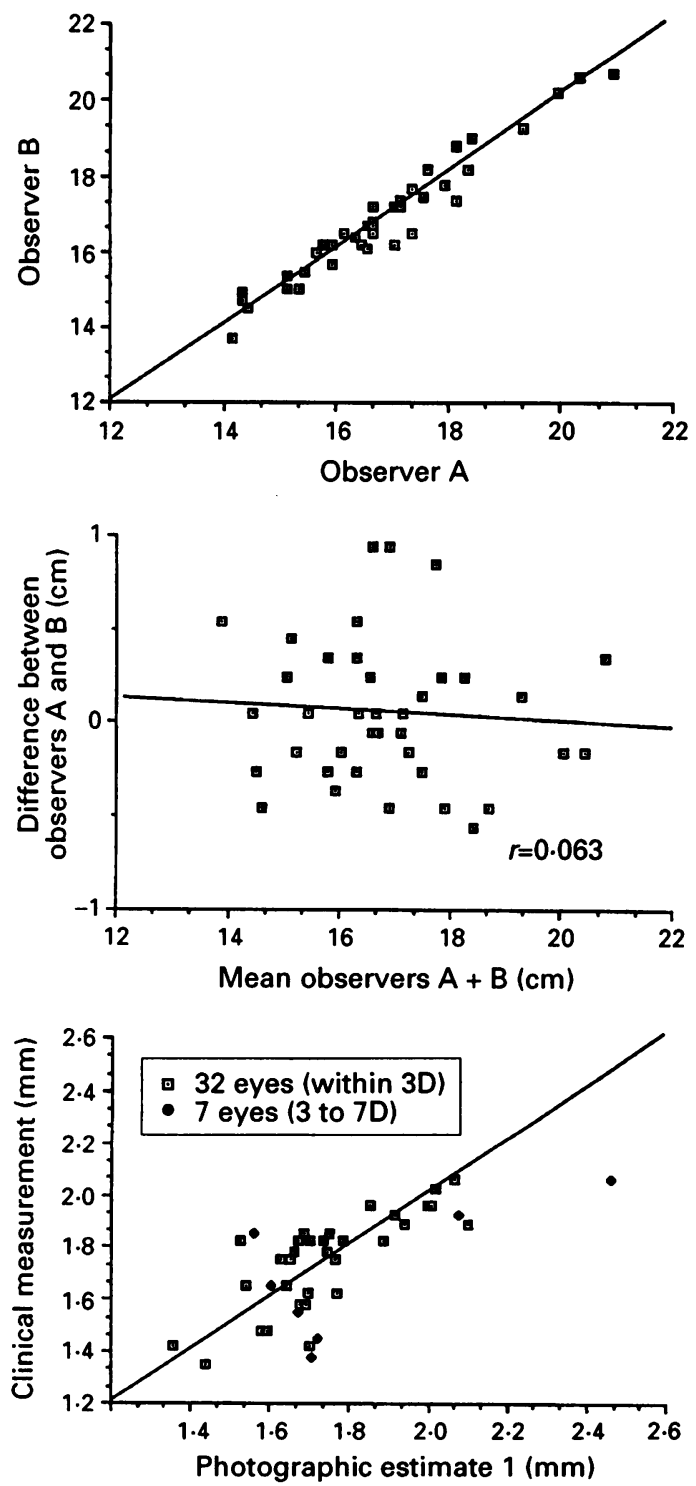

against the mean (Fig 2). This method of assessing agreement between two methods of measuring the same feature was described by Altman and Bland. ${ }^{11}$ Observer A did not read consistently higher or lower than observer $B$ as the slope of the regression line is not significant. The mean of the measurements was therefore used in further calculations. The optic discs measured from $1.365-2.388 \mathrm{~mm}$, mean $1.717(0.203) \mathrm{mm}$.

\section{COMPARISON WITH BENGTSSON AND KRAKAU'S CALCULATIONS}

Estimate 1 based on ultrasonography Magnification factor

$$
M \mathrm{e}^{1}=\mathrm{nk} /(1-\mathrm{p})
$$

$$
\begin{aligned}
\mathrm{l}= & \text { axial length }(\mathrm{m}) \mathrm{p}=\text { distance apex of } \\
& \text { cornea to principal point } 0.0016(\mathrm{~m}) \\
\mathrm{n}= & \text { refractive index } 1.336 \mathrm{k}=\text { camera } \\
& \text { constant }=0.068
\end{aligned}
$$

The results of the clinical and photographic measurements (using estimate 1) are compared in Figure 3 for the 32 eyes within $3 \mathrm{D}$ of emmetropia $(r=0.7765)$. If all the eyes are considered there is not such close correlation $(r=0 \cdot 7092)$.

\section{Estimate 2 based on spectacle refraction} Magnification factor

$$
\mathrm{Me}^{2}=\mathrm{kD} /(1-\mathrm{G} / \mathrm{D})
$$

$\mathrm{D}=58.64$ dioptre (Gullstrand) normal refractive power of the eye

$G=$ measured spectacle refraction (dioptre)

$$
\mathrm{Me}^{2}=4 \cdot 08 /(1-0 \cdot 017 \mathrm{G})
$$

The results of the clinical and photographic measurements (using estimate 2) are compared in Figure 4. Again we find a closer correlation for the 32 eyes within $3 \mathrm{D}$ of emmetropia with $r=0.8118$ and for all 39 eyes $r=0.7576$.

Estimate 3 based on refraction and keratometry Magnification factor

$$
M \mathrm{e}^{3}=\mathrm{k}\left(\mathrm{D}+\mathrm{A}+\mathrm{B} \Delta \mathrm{D}^{1}\right)
$$

$B=0.84$ (Stenstrom's calculation) coefficient of regression of $D$ on $D$

$\Delta \mathrm{D}^{1}=\left(\mathrm{D}^{1}-\mathrm{D}^{1}\right)$ and $\mathrm{D}^{1}=0 \cdot 336 / \mathrm{r}$, that is, refractive power of the cornea and

$\mathrm{D}^{1}=0 \cdot 3336 / \mathrm{r}$, that is, 'normal' refractive power of the cornea

$\mathrm{r}=$ measured corneal radius $(\mathrm{m})$ and $\mathrm{r}=0.0077 \mathrm{~m}$ (Gullstrand)

$A=$ principal point refraction of the eye $=\mathrm{G} /(1-\mathrm{G} / \mathrm{D})$

The results of the clinical and photographic measurements (using estimate 3 ) are compared in Figure 5 for 32 eyes. The best correlation between the readings is found with this photographic estimate $r=0.8614$ for the 32 eyes within $3 \mathrm{D}$ of emmetropia and $r=0 \cdot 7810$ for all 39 eyes.

There is a significantly greater difference between the photographic and clinical 
Figure 4 Photographic estimate 2 (Bengtsson and Krakau) versus the mean of the clinical measurements with the contact lens. The line of identity is shown.

Figure 5 Photographic estimate 3 (Bengtsson and Krakau) versus the mean of the clinical measurements with the contact lens. The line of identity is shown.

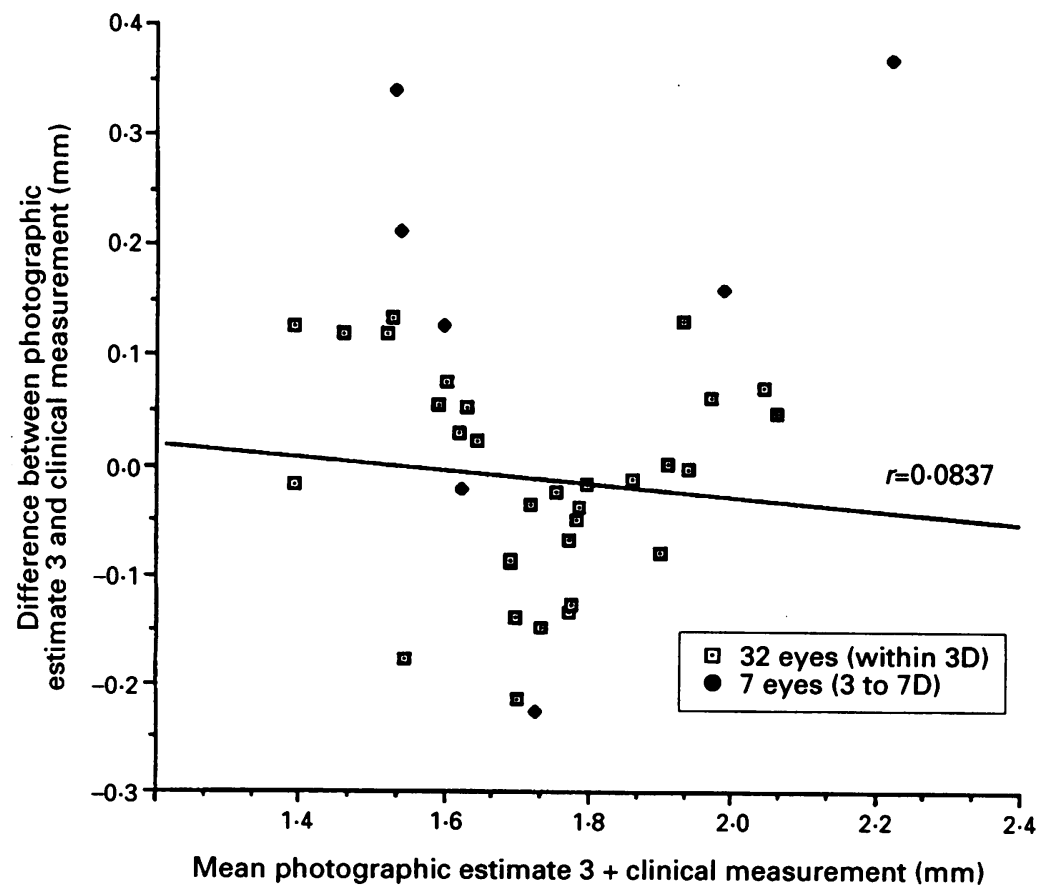

Figure 6 Difference between photographic estimate 3 and the clinical measurements versu mean of photographic estimate 3 and the clinical measurements. Simple regression line is shown for the 32 eyes within 3 D of emmetropia.
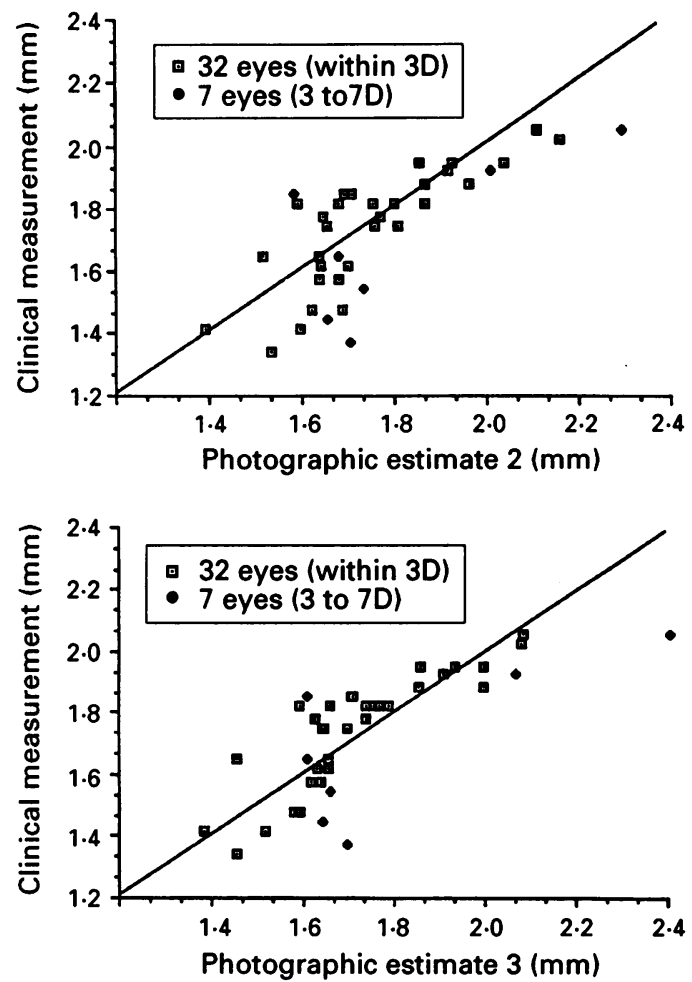

measurements for the seven eyes of greater than 3 dioptres of refractive error than for the 32 eyes within 3 dioptres of emmetropia when they are compared using a Mann-Whitney $U$ test, $p=0.0156$. This is calculated using the results obtained with estimate 3 .

Agreement was again examined by plotting the difference between the vertical disc diameter calculated by the photographic method " (using estimate 3) and the Zeiss four mirror clinical method against the mean of these two measurements (Fig 6). The mean of the clinical measurements was $1.687(0.194) \mathrm{mm}$ and that of the photographic measurements was $1 \cdot 717(0-203)$

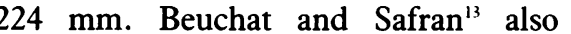
describe measuring the optic disc size at the slitlamp using a Goldmann fundus contact lens. In their method they adjusted the limits of a slit beam of light to the borders of the optic disc. They found the mean vertical diameter of 140 normal eyes to be $1 \cdot 46(0 \cdot 24) \mathrm{mm}$. No correction factor for the contact lens was calculated as they used the correction suggested by Franceschetti and $B o c k{ }^{13}$ which varies depending on the refractive error of the eye.

The magnification factor for a fundus viewing contact lens of polymethylmethacrylate with a flat front surface is $0 \cdot 925 .{ }^{14}$ However the magnifification factor for the Zeiss four mirror gonioscope is not the same as that for the Goldmann lens as the lens is manufactured using BK7 (personal communication, Carl Zeiss Oberkochen Ltd) which has a different refractive index. In fact our calculations show that it is even closer to unity. The Zeiss four mirror also has the advantage of not requiring a contact fluid.

The spot size of a certain direct ophthalmoscope has recently been advocated by Drance (oral presentation, glaucoma course, 1992) for estimating optic disc size. However this can only offer an approximation.

An adaptation of indirect ophthalmoscopy has recently been devised by Montgomery ${ }^{75}$ in 
which a transparent screen is placed at the principal plane of the condensing lens. Therefore the aerial image is located accurately and can be measured directly. The instrumentation for this technique is now available commercially..$^{16} \mathrm{~A}$ comparative study of our method and both the optic disc biometer and 78 dioptre lens, using slit-lamp biomicroscopy, is ongoing.

Different photographic techniques for quantitative analysis of the optic disc have been developed. Stereophotogrammetry measures objects in three dimensional space using stereophotographs. ${ }^{17-19}$ Cup volume can be compared with optic disc area but the accuracy of these measurements is highly dependent on the position of the camera and optic disc of the patient and the quality of the fundus photographs. ${ }^{20}$ Planimetry also examines the optic nerve head by photographs (these may be stereo to aid identification of the cup or single images) but provides only one dimensional and area measurements..$^{3-6}$ Littman $^{21}$ developed algorithms to correct for the magnification factor of the eye when using the Zeiss fundus camera. This allows absolute values for the size of the optic nerve to be calculated. Bengtsson and $\mathrm{Krakau}^{22}$ also published a formula for the magnification of the Zeiss fundus camera. Mansour $^{23}$ compared horizontal disc diameter calculated from fundus photographs using Bengtsson and Krakau's formula and the trigonometric curves described by Littman. Mansour found that Bengtsson and Krakau's formula was an acceptable approximation of Littman's formula which used anterior corneal curvature and the refractive power of the eye although it underestimated by $0.9 \%$. More recently Bengtsson and Krakau ${ }^{10}$ published their corrections for different fundus cameras.

All the above methods (and ours) are reliant on the observer's interpretation of the optic disc boundary. Those using photographs also require time consuming analysis after the patient has left the outpatient department. Computerised image analysis yields topographic maps from stereophotogrammetry (for example, Humphrey retinal analyser ${ }^{24}$ or Rodenstock optic nerve head analyser ${ }^{25}$ ) or confocal scanning laser ophthalmoscopy $^{8}$ (Heidelberg retina tomograph) but is also reliant on the accuracy of boundary recognition. It may give more 'instant' results but such technology is not readily available for most clinicians. Projecting multiple beam interference fringes onto the retina ${ }^{26}{ }^{27}$ has been shown to allow accurate measurement of fundus structures but this technique is, at present, a research tool.

The vertical disc diameter obtained by our clinical method shows good agreement with the planimetric photographic techniques. The intraobserver variation is within acceptable limits and demonstrates the repeatability of this technique. The best agreement was obtained with estimate 3 and the clinical method $(r=0 \cdot 8614)$. This is comparable with that achieved comparing optic disc area measured with the Rodenstock optic nerve head analyser and manual planimetry. ${ }^{28}$ The measurements we made were 'rounded up' or down to the nearest $0.1 \mathrm{~mm}$ and this may have reduced the agreement with the photographic method. It is possible to measure to $0.05 \mathrm{~mm}$ on the slit beam scale. Photographic methods have inaccuracies despite attempts to correct for ametropia. Pach et $a l^{20}$ showed that decentration of a retinal object and axial myopia enlarged image size. Arnold et al $^{29}$ developed a model eye to assess this with the Zeiss fundus camera. They show that the measured magnification varies from calculated formulas and that axial myopia and hypermetropia caused a magnification change of $-24 \cdot 63 \%$ to $+18 \cdot 1 \%$. Lotmar ${ }^{30}$ showed that the camera to eye distance varies the magnification of the image in ametropia. Such errors may have reduced the agreement we found between the planimetric and clinical methods. The closer correlation found when eyes within 3 dioptres of emmetropia were evaluated would support this. This does imply that Bengtsson and Krakau's calculations do not deal adequately with greater ametropia as the above authors found with Littman's corrections. For the seven eyes with higher refractive errors the photographic measurements tended to be larger than the clinical measurements with a significantly greater scatter than that seen for the 32 eyes within 3 dioptres of emmetropia. This clearly needs further investigation with a greater range of refractive errors.

There are a number of points to bear in mind when performing the technique. The contact lens must be kept vertical throughout the measurement as tilt may introduce inaccuracy in the measurement taken. We noted that a tilted lens produced a parallelogram of light rather than a rectangle. A bright but very narrow beam made it easier to match the height of the beam with the vertical disc diameter.

This clinical method is a simple technique for use at the slit-lamp biomicroscope during routine examination of the optic nerve head. It allows a very rapid evaluation of optic disc size and little if any recalculation is required. Disc size alone may be of value in determining the presence of optic nerve hypoplasia or a colobomatous large disc. In addition, in a glaucoma suspect, the same lens facilitates examination of the retinal nerve fibre layer and provides a stereoscopic image of the optic cup. This information, in conjunction with optic disc dimensions, assists the ophthalmologist in evaluating whether the size of the optic cup is appropriate for the size of the optic disc or if glaucomatous damage should be suspected. We recommend optic disc measurement with Zeiss four mirror contact lens as part of the routine initial examination of the glaucoma suspect.

We wish to thank Mr Ahmed Sadiq for his help with measurement of the vertical optic disc diameter from photographic slides, and $\mathrm{Mr}$ Peter Pawson for his help with clinical data collection.

1 Jonas JB, Gusek GC, Naumann GOH. Optic disc morphometry in chronic primary open-angle glaucoma I Morphometric intrapapillary characteristics. Graefes Arch Clin Exp Ophthalmol 1988; 226: 522-30.

2 Quigley HA, Brown AE, Morrison JD, Drance SM. The size and shape of the optic disc in normal human eyes. Arch Ophthalmol 1990; 108: 51-7.

3 Jonas JB, Gusek GC, Guggenmoos-Holzmann I, Naumann GOH. Variability of the real dimensions of normal human optic discs. Graefes Arch Clin Exp Ophthalmol 1988; 226:

4 Bengtsson $B$. The variation and covariation of cup and disc diameters. Acta Ophthalmol 1976; 54: 804-18.

5 Balazsi AG, Drance SM, Schulzer M, Douglas GR. Neuroretinal rim area in suspected glaucoma and early chronic open-angle glaucoma. Arch Ophthalmol 1984; 102: $1011-4$

6 Jonas JB, Gusek GC, Naumann GOH. Optic disc, cup and neuroretinal rim size, configuration and correlations in normal eyes. Invest Ophthalmol Vis Sci 1988; 29: 1151-8. 
7 Montgomery DMI. Measurement of optic disc and neuroretinal rim areas in normal and glaucomatous eyes - a new retinal rim areas in normal and glaucomatous

8 Cioffi GA, Robin AL, Eastman RD, Perell HF, Sarfarazi FA, Kelman SE. Confocal laser scanning ophthalmoscope reproducibility of optic nerve head topographic measurements with the confocal laser scanning ophthalmoscope Ophthalmology 1993; 100: 57-62.

9 Elkington AR, Frank HJ. Clinical optics. Oxford: Blackwell Scientific, 1984: 154-5.

10 Bengtsson B, Krakau CET. Correction of optic disc measurements on fundus photographs. Graefes Arch Clin Exp Ophthalmol 1991; 230: 24-8.

11 Bland JM, Altman DG. Statistical methods for assessing agreement between two methods of clinical measurement. agreement between t
Lancet 1986: 307-10.

12 Franceschetti A, Bock RH. Megalopapilla: a new congenital anomaly. Am f Ophthalmol 1950; 33: 227-34.

13 Beuchat L, Safran AB. Optic nerve hypoplasia: papillary diameter and clinical correlation. $\mathcal{f}$ Clin Neuro-ophthalmol 1985; $5: 249-53$.

14 Shields MB, Tiedemann JS. Binocular ophthalmoscopic techniques for evaluation of the optic nerve head. In: Varma $R$, Spaeth GL, eds. The optic nerve in glaucoma. Philadelphia: Lippincott, 1993; 137-46.

15 Montgomery DMI. Clinical disc biometry in early glaucoma. Ophthalmology 1993; 100: 52-6.

16 Pyott AAE, Montgomery DMI. Inter-observer variation in clinical optic disc biometry. Eye 1993; 7: 452-6.

17 Takamoto T, Schwartz B. Reproducibility of photogram metric optic disc cup measurements. Invest Ophthalmol Vis Sci 1985; 26: 814-7.

18 Schwartz B. New technique for the examination of the optic disc and their clinical application. Trans Am Acad Ophthal mol Otolaryngol 1976; 81: 227-35.

19 Portney GL. Photogrammetric analysis of volume asymmetry of the optic nerve head cup in normal, hypertensive and glaucomatous eyes. Am $\mathcal{F}$ Ophthalmol 1975; 80: 51-5.
20 Pach J, Pennell DO, Romano PE. Optic disc photogrammetry: magnification factors for eye position, centration, and ametropias, refractive and axial; and their application in the diagnosis of optic nerve hypoplasia. Ann Ophthalmol 1989; diagnosis of

21 Littmann $\mathrm{H}$. The determination of the true size of objects in the background of the living eye. Klin Monatsbl Augenheilkd 1982; 180: 286-9.

22 Bengtsson B, Krakau CET. Some essential optical features of the Zeiss fundus camera. Acta Ophthalmol 1977; 55: 123-31.

23 Mansour AM. Measuring fundus landmarks. Invest Ophthalmol Vis Sci 1990; 31: 41-2.

24 Dandona L, Quigley HA, Jampel HD. Variability of depth measurements of the optic nerve head and peripapillary retina with computerized image analysis. Arch Ophthalmol retina with compute

25 Caprioli J, Klingbeil U, Sears M, Pope B. Reproducibility of optic disc measurements with computerized analysis of stereoscopic video images. Arch Ophthalmol 1986; 104: 1035-9.

26 Kennedy SJ, Schwartz B, Takamoto T, Eu JKT. Interference fringe scale for absolute ocular fundus measurement. Invest Ophthalmol Vis Sci 1983; 24: 169-74.

27 Baumbach P, Rassow B, Wesemann W. Absolute ocular fundus dimensions measured by multiple-beam interference fringes. Invest Ophthalmol Vis Sci 1989; 30: 2314-9.

28 Mikelberg FS, Douglas GR, Schulzer M, Airaksinen PJ, Wijsman K, Mawson D. The correlation between cupdisk ratio, neuroretinal rim area, and optic disk area measured by the video-ophthalmograph (Rodenstock measured by the video-ophthalmograph (Rodenstock analyzer) and

29 Arnold JV, Gates JWC, Taylor KM. Possible errors in the measurement of retinal lesions. Invest Ophthalmol Vis Sci 1993; 34: 2576-80.

30 Lotmar W. Dependence of magnification upon the camera-toeye distance in the Zeiss fundus camera. Acta Ophthalmo $1984 ; 62: 131-4$. 